\title{
Excellence in design for online business education
}

\author{
Annora Eyt-Dessus and Leonard Houx \\ University of London
}

\begin{abstract}
The Business School is one of the UK's top ranked business schools, renowned for its expertise in Finance in particular, and its location in the heart of London. The School has been steadily expanding for more than a decade but was reaching the limits of its physical campus space. However, there remained the desire to develop innovative learning, expand and reach new audiences. Developing a new distance program, that built on the School's existing reputation for Finance, showcased its world-class faculty and had a global reach was identified as the ideal opportunity to explore a new strategic direction. We built a fully online program with a high rate of engagement, satisfaction and achievement. Its enrolment continues to grow, increasing our international diversity. Online learning is now embedded as a core part of the School's strategy and is seen as key to future expansion. Beyond this, our organisation cultivated far more expertise and best practice to draw on in a time of crisis than if we had outsourced. This shows the high impact an excellent learning design can make to the culture and capacity of an organisation.
\end{abstract}

Keywords online learning; distance learning; learning design; innovation; business; finance, business education

\section{Choosing online distance learning}

The Business School is one of the UK's top ranked business schools, renowned for its expertise in Finance in particular, and its location in the heart of London. The School has been steadily expanding for more than a decade but was reaching the limits of its physical campus space. However, there remained the desire to develop innovative learning, expand and reach new audiences. Developing a new distance program, that built on the School's existing reputation for Finance, showcased its world-class faculty and had a global reach was identified as the ideal opportunity to explore a new strategic direction.

It was decided that we would develop a two year, 15-hour per week MSc in our flagship subject, finance, with a small in-house two-person team. It was an ambitious project, that some might have assigned to an external development company. But we knew developing our own online MSc could do a lot for us. Beyond expanding the reach of our brand, it would show innovation in teaching practice and help the School build and show its own capability for online teaching. It would help our staff teach better online, and possibly even help them to further develop their face-to-face approaches.

\section{Aligning design to learning (and teaching) needs}

In order to ensure that our program was a success we carefully considered the learning needs of our prospective students, as well as those of our teaching staff. With relatively limited time and resources we wanted to ensure that both the program, and learning design, were well aligned and would reduce barriers to adoption, interaction and success.

\section{Students: busy, international, needing support}

The students we were recruiting would need support. We expected our online students to be older (25+), more international, and busier with substantial work and family commitments (Jaggars, 2013). So they would have less time, and familiarity with academic standards.

At the same time, they would be paying over $£ 20,000$ for a degree from a top-ranking business school. Whilst some might expect it to be easy as it was online and part-time, all would be right to demand a high level of support.

It appeared clear that a typical UK Higher Education pedagogical approach - resource-heavy, inconsistent, with limited feedback - would not meet our students' expectations and needs. Letting students study as they wished, 
in a haphazard and unstructured way, based on a limited understanding of the undertaking of a part-time distance MSc, would not be a well-informed strategy (Kirschner et al., 2013). Rather, their needs would be best met by a design focussed on feedback, motivation and support.

Our program design therefore reflects these considerations. We kept the course structure simple and consistent. Students only study one module at a time, one lesson a week, lasting seven weeks - the first six for teaching, the seventh for assessment. Most activities are asynchronous, with a live one-hour session each week, making it easier for students across continents to progress at their own pace, but also fit in time to interact with staff and each other.

\section{Academics: busy, unconvinced, uncertain}

Teaching on this course would be finance academics from the face-to-face program. These would be many of our top academics, but few had taught a fully online module before, nor worked so closely with an instructional designer and production team. Those who had attempted online teaching previously had mixed experiences and remained unconvinced of the success of this approach. All of them already had full schedules with many other commitments. For many reasons, their expectations were not high, and they were understandably concerned about what the end product of all this effort would be like as an educational experience.

The simple and consistent structure of the modules was also chosen to benefit staff. We needed a design that would be intuitive - credible yet unburdened with jargon. It also needed to feel human - surfacing and reflecting their and their students' presence. The result was a clearly articulated sequence, designed specifically to encourage student engagement, with automated feedback built in, and explicit spaces for interaction. In addition, with an interaction designer and production team to support them, they could focus on the content itself and interactions, rather than struggle with the process of technical development.

\section{Supporting students throughout our design}

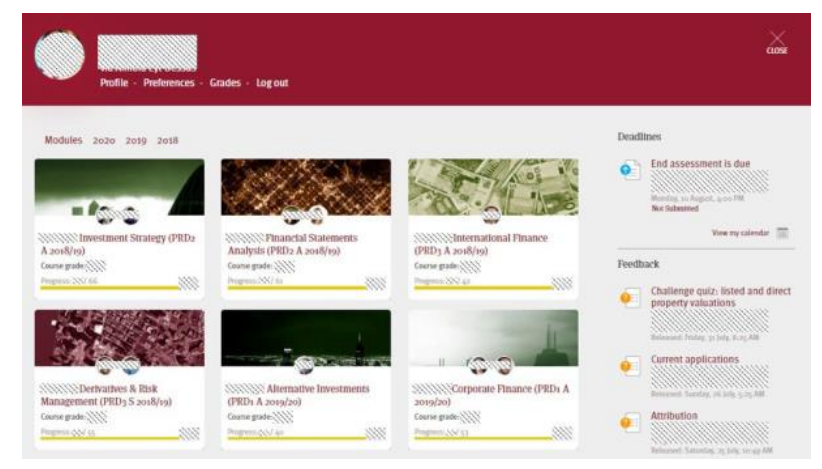

Figure 1. Student Dashboard, showing progress, deadlines and feedback

As described previously, we knew that our students might have competing priorities, had high expectations, and might have been out of study for some time. For these reasons, in addition to those accompanying a standard distance learning program, we knew that support offered to students would be key in ensuring they had a positive learning experience. We therefore designed an overlapping network of supports for our students through staff support, learning design and feedback.

\section{Dedicated staff}

Each module is supported by an academic and graduate teaching assistant. They answer questions in the forums, through email and in the weekly webinar. The program itself has a dedicated course officer who answers non-academic queries and prompts students if their participation levels have fallen. A learning technologist supports for any technical issues, and ensures these are resolved quickly to avoid unnecessary disruption to learning. 


\section{Motivation through design}

The design of activities also supports students by keeping them motivated. We design for motivation by starting simple, and building up, and tying together learning content and activity elements. By also releasing each week's learning activities gradually, we supported student to stay focused and motivated, rather than releasing everything at the start of the module which might be overwhelming and daunting. The design also aimed to motivate students through continued feedback throughout the module, building a sense of reciprocity and progression.

\section{Feedback via tutor, peer, self and automated responses}

Lastly, we supported students, not just through automated feedback, but with a variety of different methods and social interactions. Students receive peer, automated and academic feedback in each session, some building on prior layers of feedback.

\section{Designing to encourage and reinforce learning}

\section{Constraints and optimisation of our learning environment}

In building the online MSc, we faced some considerable constraints. We were constrained to the existing technology infrastructure. None of it - platform, video hosts, webinar software - could be changed. And none of it had been procured for distance learning. Nevertheless, our existing technology infrastructure was rich enough that we could build on top of it and provide a consistent, seamless, data-rich learning experience.

We used our institutional Moodle platform and plugins, but applied a new custom theme to it, aligned with our learning design and student online learning needs. We put a lot of effort into this environment, to ensure that our learners had a clear sense of the sequence, that navigation was intuitive, efficient and seamless and that they spent their time and energy in the LMS learning and not wasting time trying to understand it.

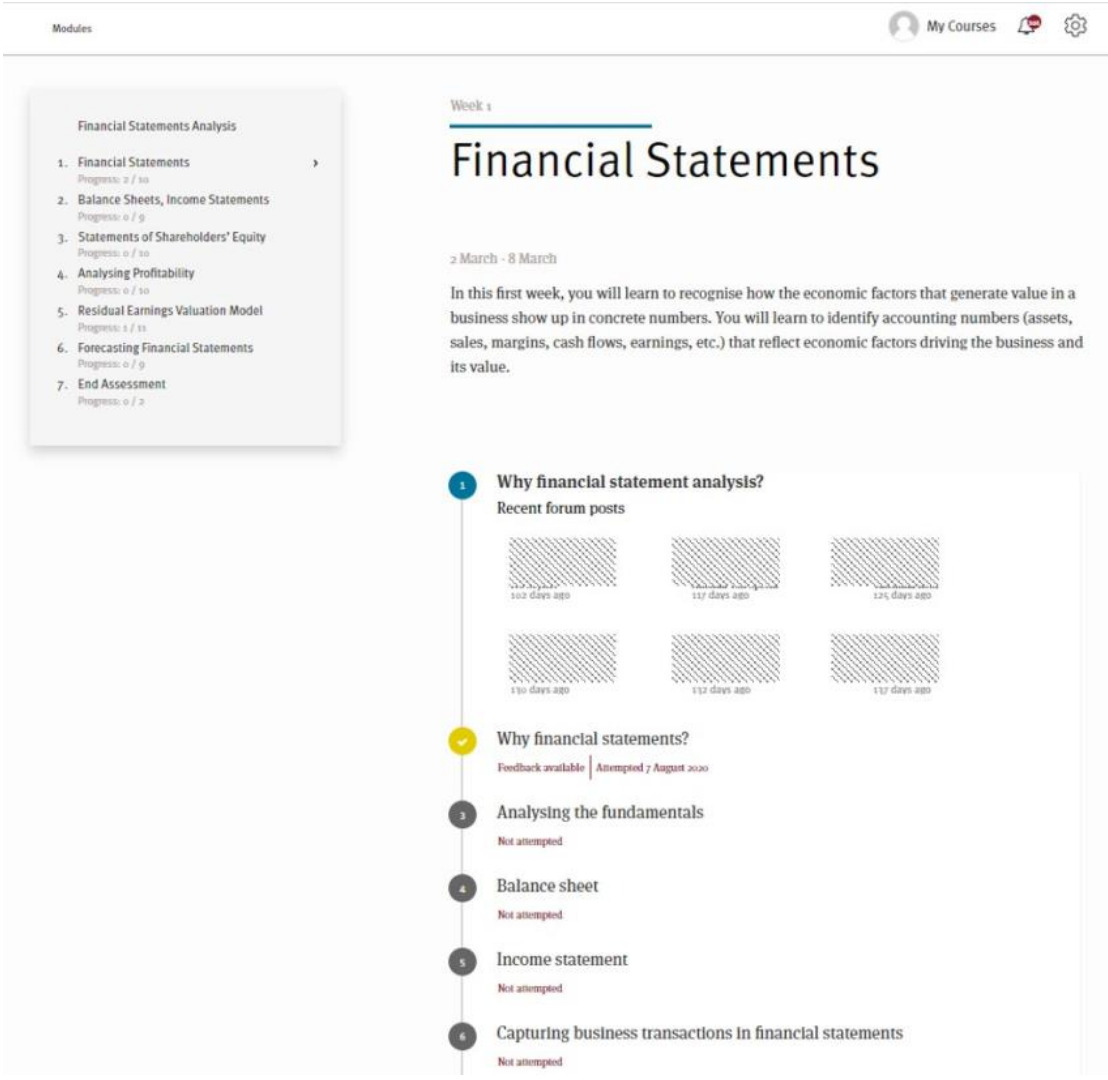

This was done by:

Figure 2. Example of a weekly topic with customised theme 
- Removing unimportant or redundant information

- Making important signposting information clearer

- Moving important signposting to the pages where it is most relevant

- Giving clearer, more immediate feedback on task completion

- Making the navigation process more logical

- Removing unnecessary steps in the navigation process

We also ensured that activity completion was on for all activities, so students could get a sense of their progress, and to help staff monitor engagement and offer support. This was enhanced in the theme by creating a modal pop up when activities have been completed, giving helpful system feedback and encouraging students to keep going.

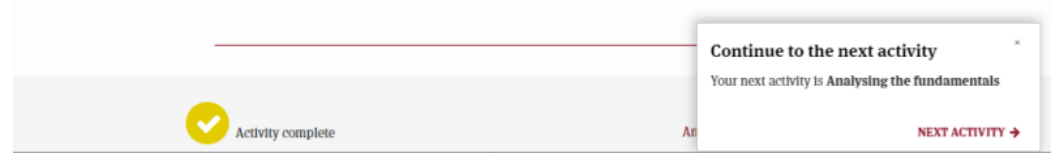

Figure 3. Custom theme development - signposting and encouraging flow

Being aware that many of our students might be juggling other commitments and be learning 'while on the go' we also ensured that this theme worked well on mobile devices, without the need to install an app or similar. Instructional design sequence

We kept the course structure simple. Students study one module at a time, one lesson a week, lasting seven weeks - the first six for teaching, the seventh for assessment. Within that, we established an activity sequence, that ran consistently each week. This consists of five parts.

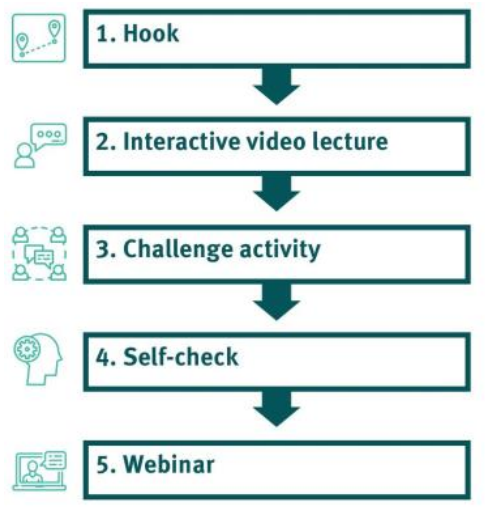

Figure 4. Activity sequence for online MSc

In several ways at once, this activity sequence ensured better learning. It ensured students could manage their time more easily because they would quickly learn the sequence and recognise the work they would need to for the week. It ensured we could include best practice more consistently and that we could, with an understanding of the overall pattern, also include an interesting mix of activities and media types.

The consistency of the activity sequence ensured our media and LMS developers could become proficient in developing these media and activity types. Because the activity sequence was carefully designed to increase in complexity and layer on feedback, the media and activity types can build on each other, making each lesson more than the sum of its parts.

\section{Embedding interactivity for learning}


Within this structure, we designed interactivity designed to build students' engagement, practice level, and feedback.

Interactivity for engagement

We designed for engagement by starting simple and building up. For example, in the first activity, we start with a lightweight discussion question. We also designed for engagement by tying together learning elements. For example, we couple each video with a quiz. This pattern, research has shown, increases learner attention (Szpunar et al., 2013). The 'challenge activity', often a bigger piece requiring application of the theory covered in the lectures, ties in by building on previous learning.

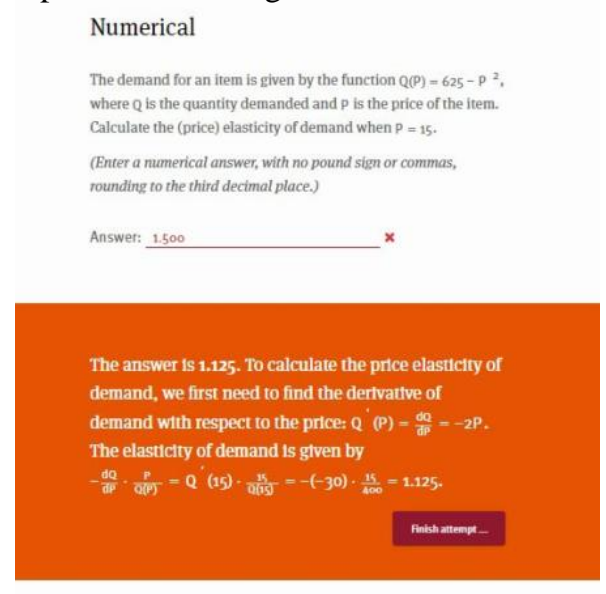

\section{Figure 5. Example of feedback given in video lecture quiz}

Lastly, drawing on research showing that this increases engagement, and creates a sense of flow (Choi et al., 2007; Skadberg et al., 2004), we ensured that immediate automated feedback was present throughout the student journey, as detailed below.

Interactivity for building long-term memory and complex skills

We designed for long-term memory and complex skills. To build long term memory, we created challenging quiz questions take advantage of the 'testing effect'. To build complex skills, we created 'challenge activities' that would challenge students to synthesise their learning thus far. These could be, for example, a simulation, an analysis from a data set, or short essay questions.

\section{Interactivity for building feedback}

Lastly, we focused on building a rich variety of types of overlapping feedback the student would receive through each lesson. Students receive social (peer-to-peer), automated and academic feedback in each session, some of which is building on previous feedback, or giving students an interactive way to process feedback. The self-check allows them to reflect, and gives direction to the live webinar session where teaching staff feedback on student questions about the learning content.

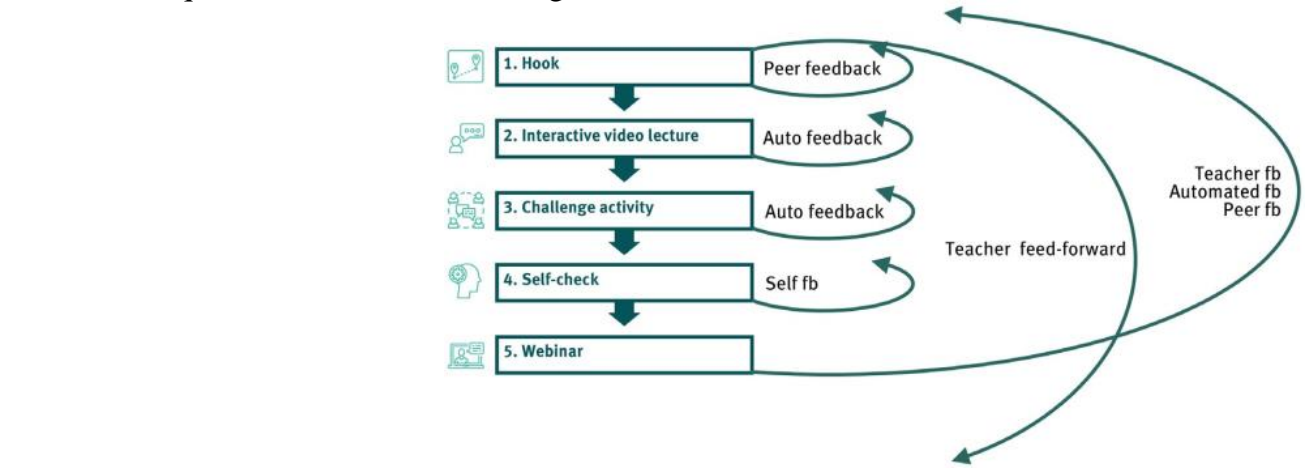

Use of media elements

Figure 6. Activity sequence for online MSc with feedback types 
Multimedia is a key part of the online MSc. Students appreciate video because it gives them independence and control of the content and because it a familiar medium for them (Scagnoli et al., 2019). It also showcases our academics, making them visible and audible to students, even when they are working asynchronously. It created a sense of presence in what can often be a 'distant', rather than just 'distance', learning experience, and which we know can be important for learning (Mayer, 2014). Lastly, it reassured academics that they were very much a key part of teaching, even if online.

Each weekly topic comprised around one hour of video 'lecture' content. However, noting research on video length, these were broken down into smaller 15-minute sections. We worked with the academics to help them learn about Mayer's cognitive theory of multimedia principles: particularly segmentation, multimedia, and redundancy (2017). We also emphasised that users scan, giving another reason to reduce text.

We were however conscious that many of our students might not be native English speakers, or might have other special educational needs. Therefore, each of our video lectures is accompanied by a PDF transcript, which our students - even those without EFL or SEN - have confirmed they use and find useful as companion material when watching the videos. With further updates to our institutional video platform we are now also adding captions to all of our media.

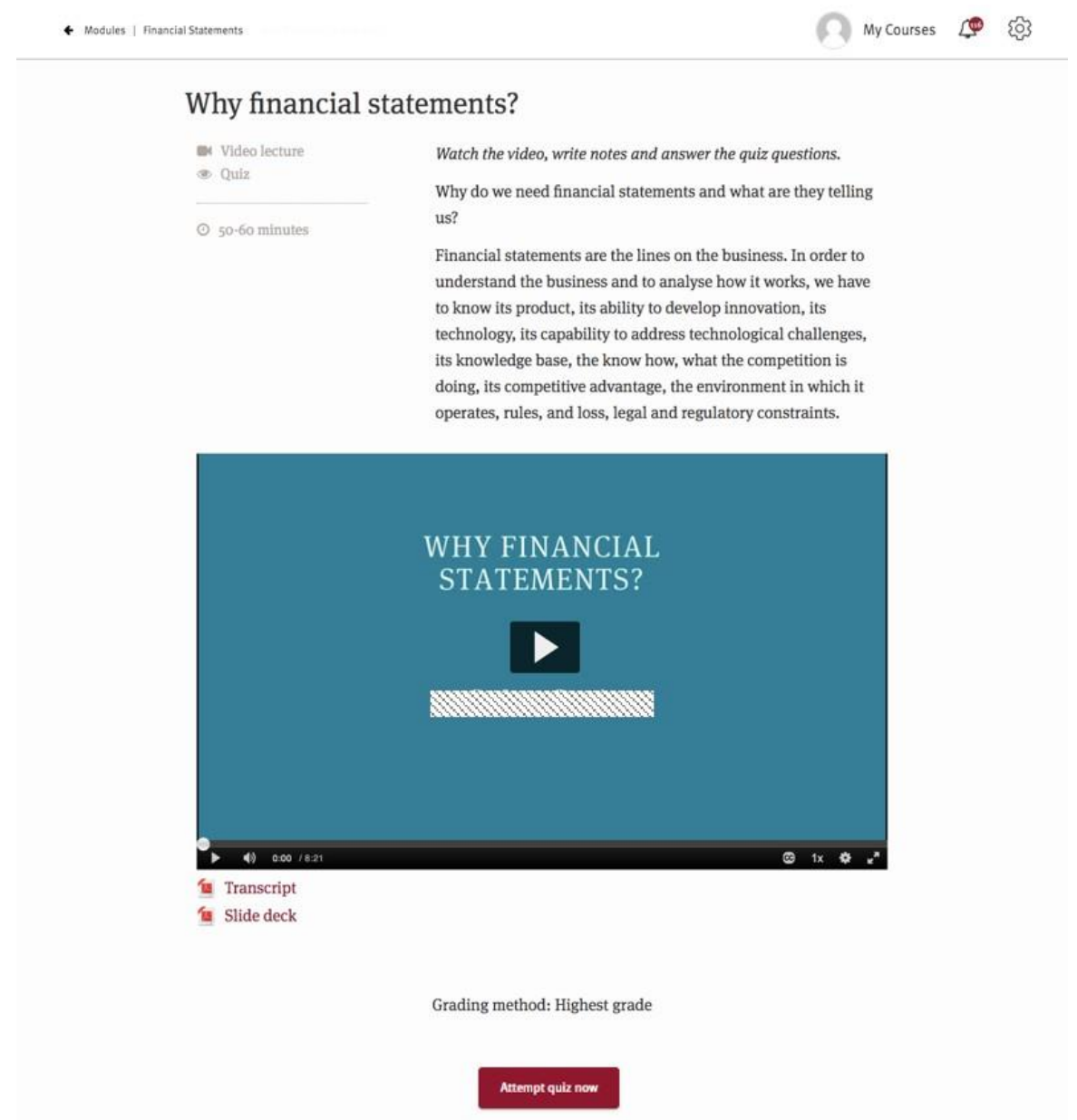

Figure 7. Example video lecture page, showing transcript and link to quiz

Another area where we spent particular time was in the introductory or 'hook' videos, recorded as a piece to camera, which were written to tell a story to gain attention, show relevance and pose a challenge or question methods shown to increase learning and engagement. The ability for students to see and hear teaching staff in the video also strongly enhances the sense of social and teaching presence (Jones et al., 2008), which we know students appreciate, and again puts staff front and center in the teaching environment.

\section{Supporting collaboration}


We designed collaboration between learners at key points in the lesson. We opened each week with an asynchronous forum discussion drawing on students' experience in a given area. Because our students are largely working professionals from around the world, this activity not only engaged their prior learning but gave a fascinating picture of the diversity of financial practice and experience around the world.

A reflection activity following the video lecture content allowed students to ask questions and raise points about the lesson in advance of the webinar. This, again, allowed students to learn from each other and the academic in a way that students have noted they particularly value. Academics have also noted how this discussion helps them to prepare what they discuss in the webinar.

Lastly, in the webinar, the academic collaboratively reviews the learning for the week. They might review activities, debate approaches, share news and ask student further questions.

\section{Reactions, results, and outcomes}

Overall, we have been exceptionally pleased with the results and reactions to this new development within the school. The reactions of learners and other key stakeholders to the program and learning design has been positive, and has supported good results in terms of learning satisfaction, take-up and outcomes.

\section{High engagement, high activity completion}

Participation has been excellent, with an average of $75 \%$ of non-marked activities being completed. This is $25 \%$ more than expected and $45 \%$ more than a Pareto level.

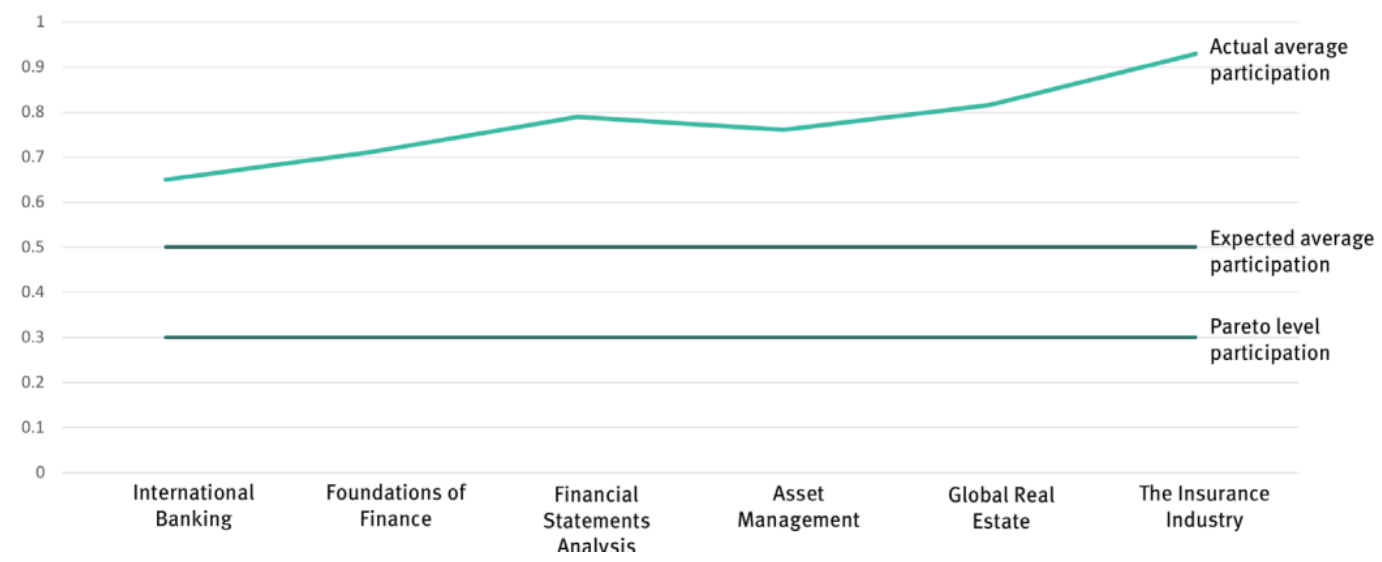

Figure 8. Activity completion rates across modules in online MSc, 2020

\section{Positive experience}

Our students rank their overall experience a solid 4.1 out of 5, the same as our overall ratings for graduate programs, and as for UK graduate programs overall (Soilemetzidis et al., 2014). 
5

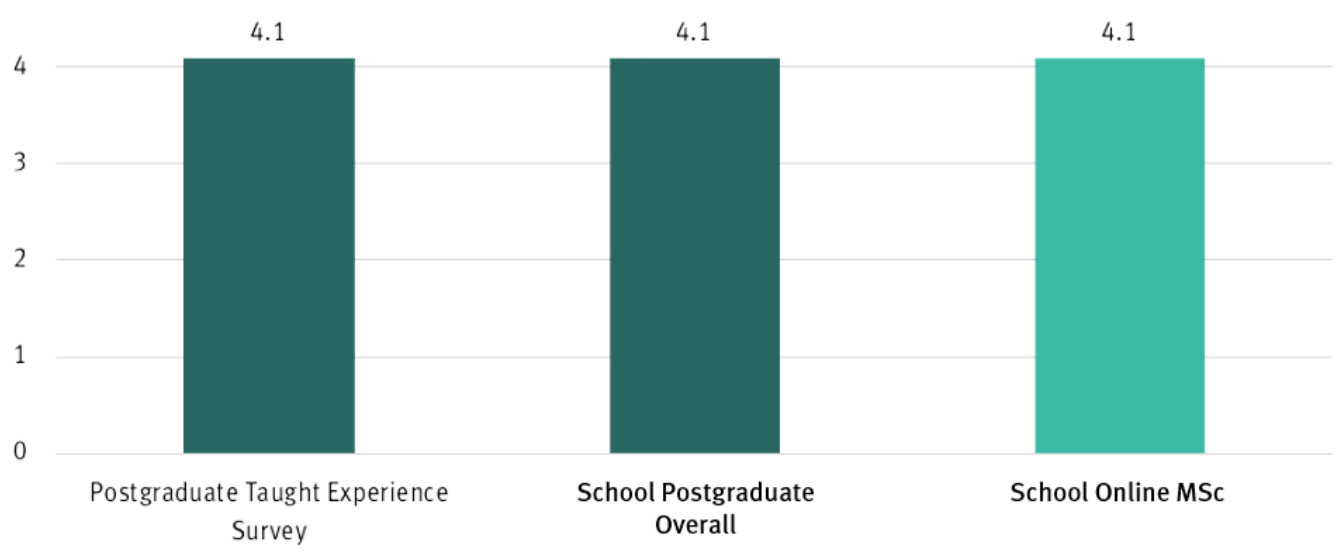

Figure 9. Course experience overall for PGTE, School PG and Online MSc

While we obviously seek to improve this year on year, it is still a reassuring counter to the myth that fully online learning is by nature 'not as good' as face-to-face or blended approaches.

\section{Strong academic achievement}

It is hard to accurately compare marks of our students with those in other graduate courses, as the assessments and timelines are too different. We are however proud to see that the design we have created supports students to achieve good outcomes, and that their method and means of study seems to give them no disadvantage.

0.5

0.4

0.3

0.25

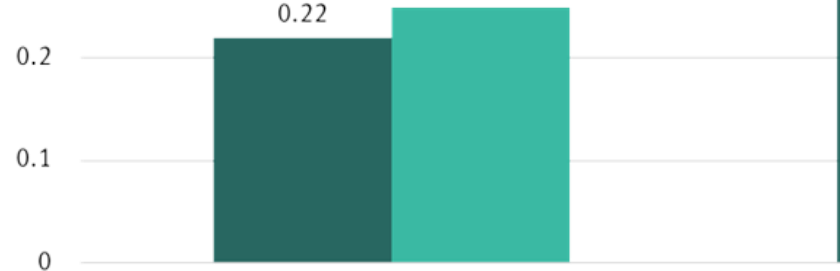

Merits

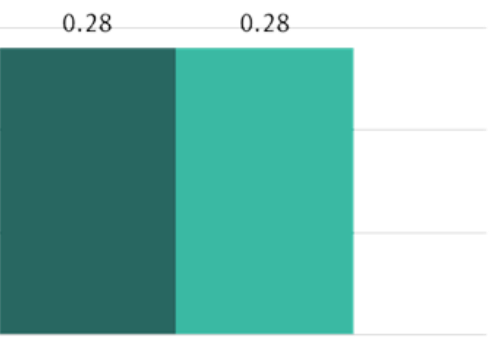

Distinctions

- All other MSc's $\quad$ School Online MSc

Figure 10. Merits and Distinctions for all School MSc's and Online MSe

\section{High retention}

The MSc has excelled at motivating and retaining our students. Distance learning degree programs have notoriously high dropout rates (Bawa, 2016). Our retention, however, has been $94 \%$. 


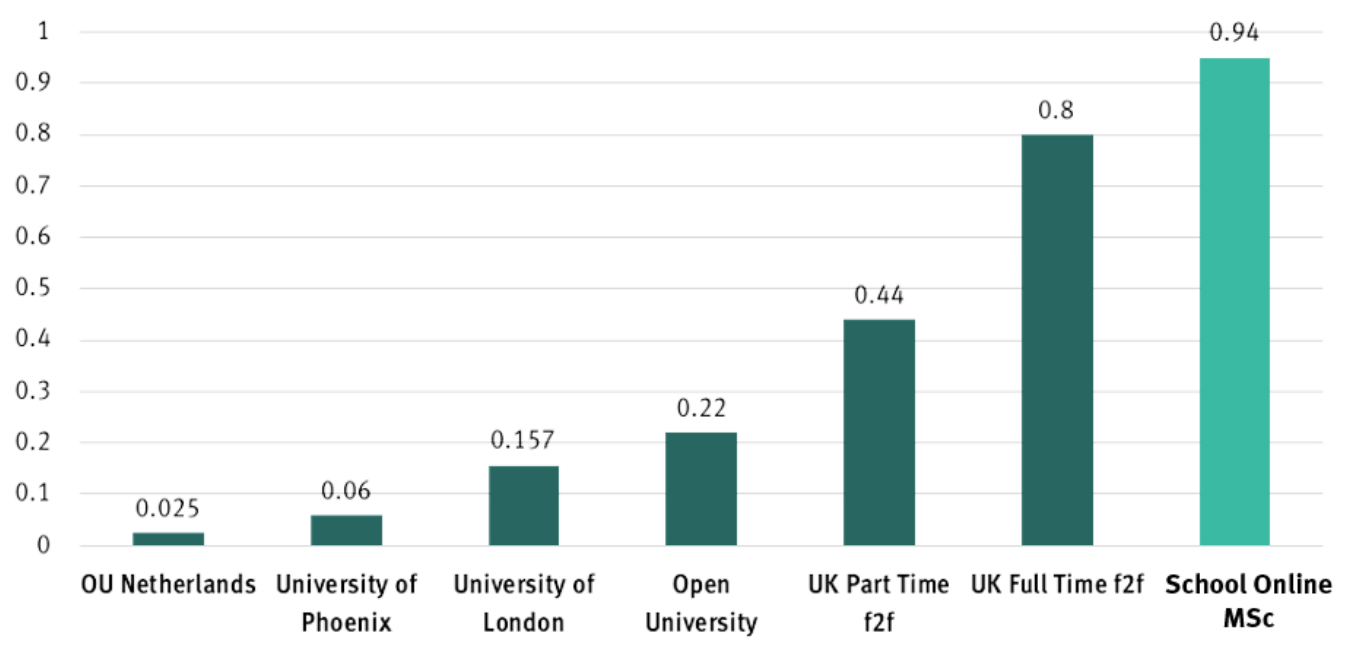

Figure 11. Retention rates across other distance learning degrees and Online MSc

\section{More digitally confident academics}

Lastly, the MSc succeeded as capacity and capability building for our teachers. Our teachers agreed that having taught and developed a module, they now felt confident teaching online (4.65/5). And it had helped their emergency teaching (4.5/5). It even, they agreed, helped inform their face-to-face teaching.

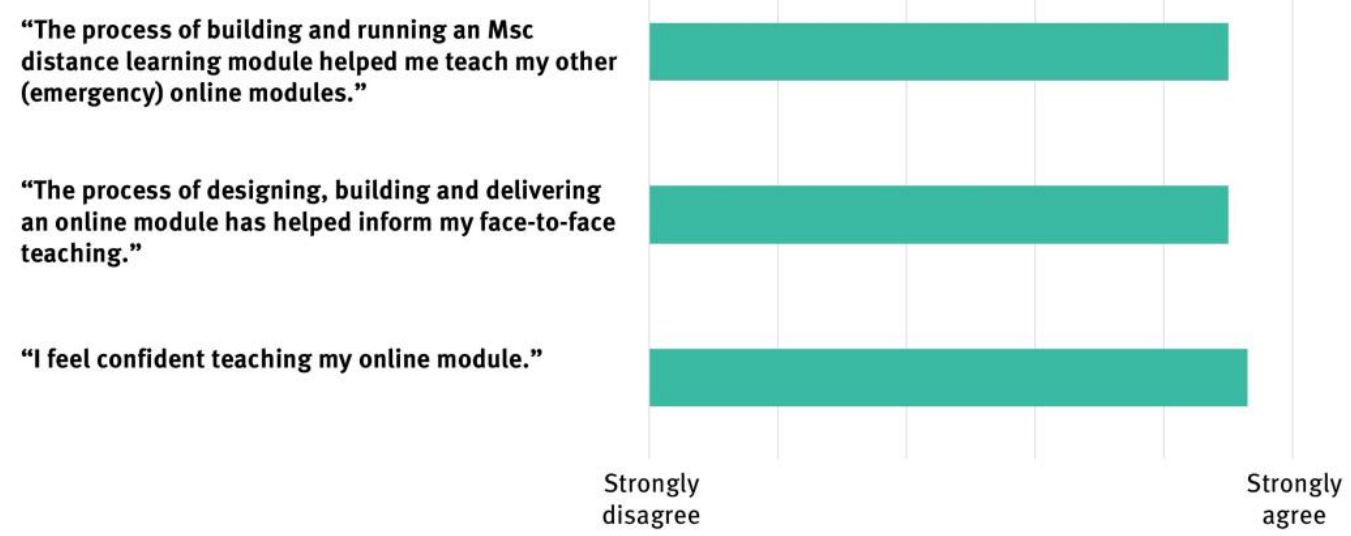

Figure 12. Responses from Online MSc academics, regarding impact on their teaching

This benefit was studied by Roblyer as a "reverse impact" phenomenon (2009). She found 75\% of academics agreed online teaching helped inform their face-to-face teaching, but responses from our academics teaching on the online MSc have even exceeded this impact (4.5/5).

\section{Advancing and innovating practice}

During the development of this course, we customised our online learning environment to bring a higher level of focus. We took great care in making all navigation and signposting efficient and meaningful. We innovated with a clearer, more efficient sense of place and progression. We designed messages, visuals and interactions that indicated place, direction and movement. This is why, for example, LMS Pulse wrote about the design as 'revolutionary' (Duque, C., 2016).

In addition, we innovated with a detailed activity sequence that worked in an environment that can sometimes be seen as inhospitable to instructional design. Familiar and intelligible enough to win adoption, yet different enough to challenge establish practice and ensure best practice. Simple enough to internalise, yet (drilling down) richly underpinned with research. Flexible enough to allow staff to bring their own ways of teaching to the fore, but consistent enough for students to find their rhythm. 
Lastly, we innovated with substantial cultural and organisational change in the business school. Through the MSc, the School has become confident we can deliver online learning. So much so that we have launched an online MBA and the Digital learning team has tripled in size. It also formed the foundation from which we delivered our emergency teaching with our team supporting the building of teaching modules, and online MSc teaching staff sharing their experiences and mentoring other academics in teaching online.

\section{Conclusions and future work}

From the start we knew developing our own online MSc would be of great benefit to the school. We built a fully online program with a high rate of engagement, satisfaction and achievement. Its enrolment continues to grow, increasing our international diversity. On the back of this, we have received investment to develop another program on a larger scale. Online learning is now embedded as a core part of the School's strategy and is seen as key to future expansion.

Beyond this, our organisation cultivated far more expertise and best practice to draw on in a time of crisis than if we had outsourced the task to an external company. We believe that this clearly demonstrates the high impact an excellent learning design can make to the culture and capacity of an organisation, and hope it will inspire others to undertake similar developments.

\section{References}

Bawa, P. (2016). Retention in online courses: Exploring issues and solutions-A literature review. Sage Open, 6(1), 2158244015621777.

Choi, D. H., Kim, J., \& Kim, S. H. (2007). ERP training with a web-based electronic learning system: The flow theory perspective. International Journal of Human-Computer Studies, 65(3), 223-243.

Duque, C.T. (2016, July 1). Revolutionary fork to the Snap Moodle theme will streamline your learning workflow. LMS Pulse Retrieved from: https://www.lmspulse.com/2016/revolutionary-fork-to-the-snapmoodle-theme-will-streamline-your-learning-workflow/

Jaggars, S. S., Edgecombe, N., \& Stacey, G. W. (2013). What we know about online course outcomes. CCRC Research Overview. Community College Research Center, Columbia University.

Jones, P., Naugle, K., \& Kolloff, M. (2008). Teacher presence: Using introductory videos in online and hybrid courses. Learning Solutions Magazine, 1-10.

Mayer, R. E. (2014). Principles based on social cues in multimedia learning: Personalization, voice, image, and embodiment principles. The Cambridge Handbook of Multimedia Learning, 16, 345-370.

Mayer, R. E. (2017). Using multimedia for e-learning. Journal of Computer Assisted Learning, 33(5), $403-423$.

Roblyer, M. D., Porter, M., Bielefeldt, T., \& Donaldson, M. B. (2009). Teaching online made me a better teacher: Studying the Impact of Virtual Course Experiences on Teachers' Face-to-Face Practice. Journal of Computing in Teacher Education, 25(4), 121-126.

Scagnoli, N. I., Choo, J., \& Tian, J. (2019). Students' insights on the use of video lectures in online classes.

British Journal of Educational Technology, 50(1), 399-414.

Skadberg, Y. X., \& Kimmel, J. R. (2004). Visitors' flow experience while browsing a Web site: its measurement, contributing factors and consequences. Computers in Human Behavior, 20(3), 403422.

Soilemetzidis, I., Bennett, P., \& Leman, J. (2014). The postgraduate taught experience survey. Hesligton York: The Higher Education Academy.

Szpunar, K. K., Khan, N. Y., \& Schacter, D. L. (2013). Interpolated memory tests reduce mind wandering and improve learning of online lectures. Proceedings of the National Academy of Sciences, 110(16), 63136317.

Eyt-Dessus, A. \& Houx, L. (2020). Excellence in design for online business education. In S. Gregory, S. Warburton, \& M. Parkes (Eds.), ASCILITE's First Virtual Conference. Proceedings ASCILITE 2020 in Armidale (pp. 156-166). https://doi.org/10.14742/ascilite2020.0145 
Note: All published papers are refereed, having undergone a double-blind peer-review process.

The author(s) assign a Creative Commons by attribution licence enabling others to distribute, remix, tweak, and build upon their work, even commercially, as long as credit is given to the author(s) for the original creation.

(C) Eyt-Dessus, A. \& Houx, L. 2020 